\title{
Continuities between childhood and adult life*
}

\author{
BARBARA MAUGHAN and JULIA KIM-COHEN
}

The child, said the poet, is father of the man. As findings from long-term studies of psychopathology proliferate, so the truth of that dictum becomes ever more apparent in the mental health field. Many childhood disorders - once thought to resolve with age - are now known to cast long shadows over later development. Equally importantly, many adult disorders are now recognised as having roots in childhood vulnerabilities, traceable in some instances to the very earliest stages of development. These developmental findings have shed new light on the aetiology and course of many disorders, and underscored the usefulness of a lifespan perspective on psychopathology for researchers and clinicians alike. We focus here on three themes emerging from this rapidly expanding literature: first, evidence on the extent and complexity of childhoodadult continuities; second, some of the mechanisms proposed as mediating those links; and third, the implications that follow for nosology and clinical practice.

\section{MAPPING CHILDHOOD - ADULT CONTINUITIES}

Although hinted at for many years, the most persuasive evidence for childhoodadult continuities has come from prospective studies of unselected birth cohorts, set up by far-sighted investigators many years ago (Coleman \& Jones, 2004). As cohorts of this kind mature into adulthood, their findings offer two contrasting perspectives on links between psychopathology in childhood and in adult life. First, looking forwards from childhood, longitudinal data provide estimates of the extent to which children and adolescents with mental health

*This is one of a series of editorials being published in the Journal to mark the I0th anniversary of the Social, Genetic and Developmental Psychiatry Centre at the Institute of Psychiatry. problems are at risk of disorder later in the life course. Second, looking backwards from adulthood, they highlight early roots of adult vulnerability. Probably the most striking evidence for continuities comes from this second approach. Recent reports from the Dunedin longitudinal cohort, for example, have shown that most young adults with a psychiatric disorder had diagnosable problems much earlier in life (KimCohen et al, 2003). Of those with mental health problems at age 26 years, half had first met criteria for disorder by age 15 years; by the late teens, that figure approached $75 \%$. On this evidence, many adult disorders could be re-framed as extensions of juvenile difficulties.

Behind 'headline' findings of this kind, studies have also detailed psychopathological precursors to specific adult disorders. Some, as expected, reflect homotypic continuities: adult disorders (such as phobias) that are preceded by childhood and adolescent difficulties of a similar kind; but other developmental sequences emerging from longitudinal records are more complex. Early adult depression, for example, is commonly preceded by childhood anxiety, whereas adult anxiety is preceded by both depression and anxiety. Less predictable still are sequences (now increasingly well replicated) that link apparently quite different disorders at different stages in the life course. In the Dunedin study, for example, conduct and oppositional disorders in childhood showed expected links with later substance misuse and antisocial personality disorder; in addition, however, they were associated with increased risk of adult depression, anxiety, eating disorders, schizophreniform disorders and even mania (Kim-Cohen et al, 2003). Understanding the processes involved in these heterotypic continuities, or patterns of sequential comorbidity, constitutes a key target for future research.

With the exception of severe early-onset disorders such as autism, continuities looking forwards from childhood are typically less strong. At most half of boys with conduct disorder, for example, go on to show antisocial personality disorder (Maughan \& Rutter, 2001), and the majority of children with anxiety or depression will not have mood disorders in adult life (Wals \& Verhulst, 2005). However, longitudinal evidence also suggests that to focus on categorically defined disorders alone is to take too narrow a view. Sub-threshold levels of childhood and adolescent symptoms can signal increased risk of adult disorder, and later psychopathology is by no means the only adverse consequence of poor childhood mental health. Studies have consistently shown, for example, that many young people with early histories of psychiatric disorder go on to face problems in other aspects of their adult lives: interpersonal relationships, educational attainments and occupational functioning seem especially likely to be compromised, as also may be physical health.

\section{HOW EARLY CAN WE TELL?}

To date, most studies tracking links between childhood and adult disorders have 'begun' in middle to late childhood. However, individual differences in children's temperaments, and in behavioural styles, are evident much earlier in development: one key question thus centres on the extent to which early behaviours of this kind are also implicated in risk. Using their observations of 3-year-old children, Caspi et al (1996) documented modest but none the less conceptually meaningful associations with psychopathology at age 21 years. Behaviourally inhibited toddlers were more likely than other young children to show mood disorders in their early 20 s, and undercontrolled 3-year-olds were at increased risk of antisocial personality disorder and persistent violent offending; both groups showed higher rates of suicide attempts. Prior to age 3 years, data reaching forward to adulthood are still rare. Tracked into childhood and adolescence, however, early temperamental difficulties do show coherent associations with later mental health, although again the links are modest (Sanson et al, 2004). Many commentators conclude that very early temperamental features represent predispositions or vulnerabilities to disorder, but that later developmental processes are 
required to transform them more directly into risk (Rothbart \& Bates, 1998).

\section{MECHANISMS FOR CONTINUITY AND DISCONTINUITY}

What mechanisms underlie these longterm developmental linkages - and what contributes to discontinuities when they arise? As yet, answers to these questions are more speculative, although knowledge is increasing at a rapid pace. Key pointers have come not only from studies of individual characteristics, but also from longitudinal findings on potential risks. Here, developmental studies have highlighted associations between 'adult' disorders and both prenatal and postnatal factors that may influence neurological or psychological development. As a result, aetiological models now frequently posit interactions between individual vulnerabilities (genetic or acquired) and prenatal or postnatal environmental insults; these in turn are thought to set in train biological or psychological processes whose effects cumulate across development, or are activated by later developmental challenges. We illustrate possible pathways of this kind in relation to three disorders: schizophrenia, depression and antisocial behaviour.

Current thinking on risk mechanisms in schizophrenia illustrates the complexity of effects that may be involved (Walker et al, 2004). Largely as a consequence of insights from longitudinal studies, schizophrenia is now increasingly regarded as a developmental disorder. In part, this change of view was prompted by follow-back findings showing mild deficits in social, motor and cognitive functioning in children and adolescents, and even infants, who later went on to display psychotic symptoms. In addition, susceptibility to schizophrenia has been associated with a range of prenatal and perinatal risks (including obstetric complications, maternal stress and maternal exposure to infection in pregnancy) that have the potential to affect neurodevelopmental processes. Although direct evidence is still lacking, early insults of this kind perhaps in interaction with genetic predispositions - are thought to lay the groundwork for vulnerable neuronal circuits whose effects can compromise brain structure and function. Not all individuals with these vulnerabilities go on to show disorder; instead, final illness expression is thought to depend on later developmental processes, associated with adolescent neuromaturation or enhanced sensitivity to the effects of later stress. The role of cannabis use as one element in this constellation of risks has attracted particular attention of late (Arseneault et al, 2004).

Developmental findings have also led to new thinking about the aetiology of depression. Historically, depression was rarely diagnosed before adulthood. Developmental evidence has made clear, however, that depressive disorders are manifest in childhood and adolescence, and that the marked increase in depressive phenomena (along with the emergence of the female predominance) occurs in the early teenage years (Angold \& Costello, 2001). In addition, mood disorders are frequently associated with childhood adversity, in this case exposure to traumatic or abusive experiences in childhood, or lack of adequate parental care. Here, risks in genetically vulnerable individuals have been argued to stem from long-term effects of early stress on neuroendocrine functioning (Nemeroff, 2004), or from the psychological effects of early adversity on selfconcepts or other psychological schemas.

Aetiological models for disruptive behaviour disorders illustrate yet other insights deriving from developmental findings. Here, one long-standing puzzle centred on the heterogeneity of outcomes for antisocial young people: some face lives of continued antisocial involvement, while others achieve much better adjustment in adulthood. Developmental studies concurred with genetic findings in highlighting age at onset as a key differentiating factor here. This in turn led to the formulation of a developmental taxonomy, involving distinct aetiological models for childhoodand adolescent-onset groups (Moffitt, 1993). Adolescent-onset delinquency is in many ways normative, and is largely prompted by affiliations with deviant peers. Severe early-onset disruptiveness, in contrast, is thought to stem from interactions between early neurocognitive deficits and adverse parenting that set in train negative interactional patterns, and possibly cognitive sets, that in turn evoke reinforcing responses from others. In part, later continuities in antisocial behaviour rest on indirect chain effects, whereby poor outcomes at one stage of development elevate risks for functioning at the immediately succeeding stage. By the same token, links in the chain of risk can be broken if environmental reinforcements are disrupted. 'Turning point' experiences - lifecourse changes that offer opportunities for pro-social engagements, and provide appropriate social controls - have attracted particular attention here (Rutter, 1996). Importantly, studies have shown that processes of this kind are not confined to early stages in development, but can arise well into adult life.

\section{IMPLICATIONS}

As this brief overview suggests, developmental perspectives have led to important reconceptualisations of disorder in a number of domains. At this stage, however, these insights have yet to be incorporated into classification systems, and diagnostic criteria continue to place most weight on current symptomatology. Yet for Kraepelin (1919), information on the overall course of a disorder was at least as important for diagnostic decision-making as the presence of specific behavioural indicators. Evidence from longitudinal studies increasingly supports the view that future revisions of the diagnostic criteria should include information on individuals' lifetime experience of psychopathology alongside details of current symptoms (Widiger \& Clark, 2000). For the clinician, these findings also underscore the usefulness of a developmental perspective. Many patients seen in adult services will already have experienced episodes of disorder, or been subject to early stress; many child and adolescent patients will face long-term vulnerabilities. Setting patients' current difficulties in the context of a longer-term view offers the promise of both more accurate diagnosis and more appropriately tailored treatments.

\section{DECLARATION OF INTEREST}

None.

\section{REFERENCES}

Angold, A. \& Costello, E. J. (200I) The epidemiology of depression in children and adolescents. In The Depressed Child and Adolescent (2nd edn) (ed. I. M. Goodyer), pp. 143-178. Cambridge: Cambridge University Press.

Arseneault, L., Cannon, M., Witton, J., et al (2004) Causal association between cannabis and psychosis: examination of the evidence. British Journal of Psychiatry, 184, $110-117$.

Caspi, A., Moffitt, T. E., Newman, D. L., et al (1996) Behavioral observations at age 3 years predict adult psychiatric disorders - longitudinal evidence from a 
birth cohort. Archives of General Psychiatry, 53, 1033-1039.

Coleman, I. \& Jones, P. (2004) Birth cohort studies in psychiatry: beginning at the beginning. Psychological Medicine, 34, 1375-1383.

Kim-Cohen, J., Caspi, A., Moffitt, T. E., et al (2003)

Prior juvenile diagnoses in adults with mental disorder. Archives of General Psychiatry, 60, 709-717.

Kraepelin, E. (1919) Dementia Praecox and Paraphrenia (transl. R. M. Barclay). Edinburgh: Livingstone.

Maughan, B. \& Rutter, M. (200I) Antisocial children grown up. In Conduct Disorders in Childhood and Adolescence (eds J. Hill \& B. Maughan), pp. 507-552. Cambridge: Cambridge University Press.

Moffitt, T. E. (1993) Adolescence-limited and lifecourse-persistent antisocial behavior - a developmental taxonomy. Psychological Review, 100, 674-70I.

Nemeroff, C. B. (2004) Neurobiological consequences of childhood trauma. Journal of Clinical Psychiatry, 65 (suppl. I), 18-28.

Rothbart, M. K. \& Bates, J. E. (1998) Temperament. In Handbook of Child Psychology, vol. 3: Social, Emotional and

BARBARA MAUGHAN, PhD, Social, Genetic and Developmental Psychiatry Centre, Institute of Psychiatry King's College London; JULIA KIM-COHEN, PhD, MRC Social, Genetic and Developmental Psychiatry Centre, Institute of Psychiatry, King's College London, UK, and University of Wisconsin at Madison, Canada

Correspondence: Dr Barbara Maughan, Box PO46, MRC Social, Genetic and Developmental Psychiatry Centre, Institute of Psychiatry, King's College London, 16 De Crespigny Park, London SE5 8AF, UK. E-mail: b.maughan@iop.kcl.ac.uk

(First received 14 February 2005, accepted 17 February 2005)

Personality Development (eds W. Damon \& N. Eisenberg) Pp. 105-176. New York: John Wiley.

Rutter, M. (1996) Transitions and turning points in developmental psychopathology: as applied to the age span between childhood and mid-adulthood. International Journal of Behavioural Development, $\mathbf{1 9}$ 603-626.

Sanson, A., Hemphill, S. A. \& Smart, D. (2004)

Connections between temperament and social development: a review. Social Development, 13, 142-170.
Walker, E., Kestler, L., Bollini, A., et al (2004) Schizophrenia: etiology and course. Annual Review of Psychology, 55, 40I-430.

Wals, M. \& Verhulst, F. (2005) Child and adolescent antecedents of adult mood disorders. Current Opinion in Psychiatry, 18, 15-19.

Widiger, T. A. \& Clark, L. A. (2000) Toward DSM-V and the classification of psychopathology. Psychological Bulletin, 126, 946-963. 